\title{
Concussion and Mild-Traumatic Brain Injury in Rural Settings: Epidemiology and Specific Health Care Considerations
}

\author{
John K. Yue Pavan S. Upadhyayula $^{3,4}$ Lauro N. Avalos ${ }^{1}$ Ryan R. L. Phelps ${ }^{1,2}$ Catherine G. Suen
}

Tene A. Cage ${ }^{6}$

${ }^{1}$ Department of Neurological Surgery, University of California San Francisco, San Francisco, California, United States

${ }^{2}$ Brain and Spinal Injury Center, Zuckerberg San Francisco General Hospital, San Francisco, California, United States

${ }^{3}$ Department of Neurological Surgery, Columbia University Medical Center, New York, New York, United States

${ }^{4}$ Department of Neurological Surgery, University of California San Diego, San Diego, California, United States

${ }^{5}$ Department of Neurology, University of Utah School of Medicine, Salt Lake City, Utah, United States

${ }^{6}$ Department of Neurological Surgery, Stanford University School of Medicine, Stanford, California, United States

J Neurosci Rural Pract 2020;11:23-33

\begin{abstract}
Address for correspondence John K. Yue, MD, Department of Neurological Surgery, University of California San Francisco, 1001 Potrero Avenue, Building 1, Rm 101, San Francisco, CA 94143 , United States (e-mail: john.yue@ucsf.edu).

Tene A. Cage, MD, Department of Neurological Surgery, Stanford University School of Medicine, Stanford, CA 94305 (e-mail: tenecage@stanford.edu).
\end{abstract}

\begin{abstract}
Keywords

- concussion

- epidemiology

- health disparity

- mild-traumatic brain injury

- rural

- prevention

- return to play
\end{abstract}

Background Mild-traumatic brain injury (mTBI) and concussions cause significant morbidity. To date, synthesis of specific health care disparities and gaps in care for rural $\mathrm{mTBI} /$ concussion patients remains needed.

Methods A comprehensive literature search was performed using PubMed database for English articles with keywords "rural" and ("concussion" or "mild traumatic brain injury") from 1991 to 2019. Eighteen articles focusing on rural epidemiology $(n=5)$, management/cost $(n=5)$, military $(n=2)$, and concussion prevention/return to play $(n=6)$ were included.

Results $\mathrm{mTBI} /$ concussion incidence was higher in rural compared with urban areas. Compared with urban patients, rural patients were at increased risk for vehicular injuries, lifetime number of concussions, admissions for observation without neuroimaging, and injury-related costs. Rural patients were less likely to utilize ambulatory and mental health services following $\mathrm{mTBI} /$ concussion. Rural secondary schools had decreased access to certified personnel for concussion evaluation, and decreased use of standardized assessment instruments/neurocognitive testing. While school coaches were aware of return-to-play laws, $\mathrm{mTBI} /$ concussion education rates for athletes and parents were suboptimal in both settings. Rural veterans were at increased risk for postconcussive symptoms and posttraumatic stress. Telemedicine in rural/low-resource areas is an emerging tool for rapid evaluation, triage, and follow-up.

Conclusions Rural patients are at unique risk for $\mathrm{mTBI} /$ concussions and health care costs. Barriers to care include lower socioeconomic status, longer distances to regional medical center, and decreased availability of neuroimaging and consultants. Due to socioeconomic and distance barriers, rural schools are less able to recruit personnel certified for concussion evaluation. Telemedicine is an emerging tool for remote triage and evaluation.
License terms

()(1) $\Theta \circledast$ 


\section{Introduction}

Mild-traumatic brain injury (mTBI) encompasses nonpenetrating TBIs with no-to-mild initial neurologic deficits. ${ }^{1}$ Concussion describes similar injuries, where linear and/ or rotational forces disrupt normal cellular activity at the gray-white junction, corpus callosum, and/or brainstem leading to disruption in consciousness. mTBIs/concussions were traditionally thought unlikely to cause longterm deficits. However, an estimated 1.7 to 5.3 million U.S. patients experience mTBI/concussion annually, ${ }^{2,3}$ with costs exceeding 17 billion dollars (U.S. dollars [USD]). ${ }^{4}$ While conventional studies quote an annual incidence of 100 to 300 cases per 100,000 people, in reality the incidence is $790 / 100,000$, while in rural areas, schools, prisons and other nonconventional locales are incorporated. ${ }^{5}$ There are some variations of how rural settings are defined internationally. In the United States, rural areas comprise open country or settlements with fewer than 1,500 residents or fewer than 999 persons per square mile ${ }^{6}$; in Canada, these numbers are 1,000 and 400 per square kilometer, respectively. ${ }^{7}$ In India, rural settlements have populations less than 5,000 or less than 400 per square kilometer, with more than $25 \%$ of male working population engaged in agricultural pursuits. ${ }^{8}$ In China, rural is generally classified as areas with less than 2,000-3,000 residents, or more populous areas with greater than $90 \%$ agricultural population. ${ }^{9}$ Generally, rural residents have decreased logistical, educational, and financial access to health care. ${ }^{10}$ Underreporting of mTBIs is attributable to decrease awareness of needing care (e.g., sports concussions), limited access to care, ineffective treatments following injury, and unclear follow-up/referral guidelines. All of which are further reduced in rural settings when compared with urban/ suburban areas. Access to neurological/neurosurgical care is limited by decreased hospital and specialty physician coverage, lack of transportation, financial constraints, and cultural attitudes. ${ }^{11,12}$ To date, management practices within rural systems of care are under-characterized from a national and international perspective.

The $\mathrm{mTBI} /$ concussion treatment is typically conservative, with a clinical diagnosis supported by neuroimaging and often a period of observation, cognitive rest, and return precautions. It has been reported that early interventions, for example, emergency department (ED) visits, may not improve outcomes in clinic compared with patients who did not seek medical treatment. ${ }^{13}$ Absence of validated treatments for acute $\mathrm{mTBI} /$ concussion does not, however, obviate the need for follow-up. It is increasingly recognized that mTBI/ concussion can cause long-term consequences, for example, functional deficits limiting return to work, and a variety of neurocognitive and neuropsychiatric symptoms. ${ }^{14-17}$ Recent studies show that up to $80 \%$ of patients may experience symptomatology and 30\% may be functionally impaired at 3 and 6 months. ${ }^{18}$ Lack of health care engagement in the setting of persistent symptoms can present significant challenges to have deleterious impacts to socioeconomic self-sufficiency and cause development of maladaptive coping mechanisms. ${ }^{19-24}$ Recovery in rural and/or low-resource settings presents additional challenges for rehabilitation and training for both patients and providers. ${ }^{23,24}$

Understanding unique considerations in rural health care utilization after $\mathrm{mTBI} /$ concussion is critical to improve access and reduce health care burden. We provide a comprehensive review of the current evidence on rural $\mathrm{mTBI} /$ concussion epidemiology, risk factors, management, and prevention efforts in rural settings for this vulnerable group of patients.

\section{Methods}

\section{Study Selection}

A literature search was performed using the PubMed database. English language articles with keywords "rural" and "concussion" or "mild traumatic brain injury" were candidates for inclusion (search criteria: "rural [title/abstract] AND (concussion [Title/Abstract] OR mild traumatic brain injury [Title/Abstract])." This search yielded 30 articles from 1991 to 2019. Study authors reviewed each article and determined its relevance to the management of $\mathrm{mTBI} /$ concussion and to urban versus rural practices. All articles were unanimously included or excluded.

Of the 30 articles, 18 were selected for inclusion ( - Fig. 1). Twelve were excluded due to inapplicability (four mixed urban/rural population without differentiation, three dental injury, one postmortem study, two non-English language, one review on mTBI, and one review on concussion law). Articles included both pediatric and adult patients.

\section{Study Analysis}

Included articles were stratified by subtopics of mTBI/concussion, including international epidemiology $(n=5)$, management and cost $(n=5)$, military and postdeployment considerations $(n=2)$, and injury prevention/return to play (RTP; $n=6$ ). Each subtopic was synthesized to elucidate issues unique to $\mathrm{mTBI} /$ concussions in rural populations, with comparisons to urban populations when available.

\section{Results}

\section{International Epidemiology of Rural mTBI}

In general, incidence of $\mathrm{mTBI} /$ concussion, proportion of motorized vehicle accidents and presence of polytrauma were increased in rural compared with urban settings. The following describes country-specific profiles (-Table 1):

Canada pediatric study: Stewart et al studied 2,112 pediatric concussions (19\% rural) from a level-1 trauma center ED in Ontario, Canada, from 2006 to $2011 .{ }^{25}$ Compared with urban, rural patients were older, had elevated ED concussion visit rates $(3.5 / 1,000$ versus $2.2 / 1,000)$, sustained 2.5 times the number of motor vehicle accidents (MVA)related concussions, were more likely have loss of consciousness (12 vs. $10 \%$ ), intracranial abnormalities (10 vs. $6 \%$ ), and admission (7 vs. $4 \%$ ). ${ }^{25} \mathrm{~A}$ higher proportion of rural children were injured in a motorized recreational vehicle (31 vs. $12 \%$ ), underscoring the need for focused concussion education in 


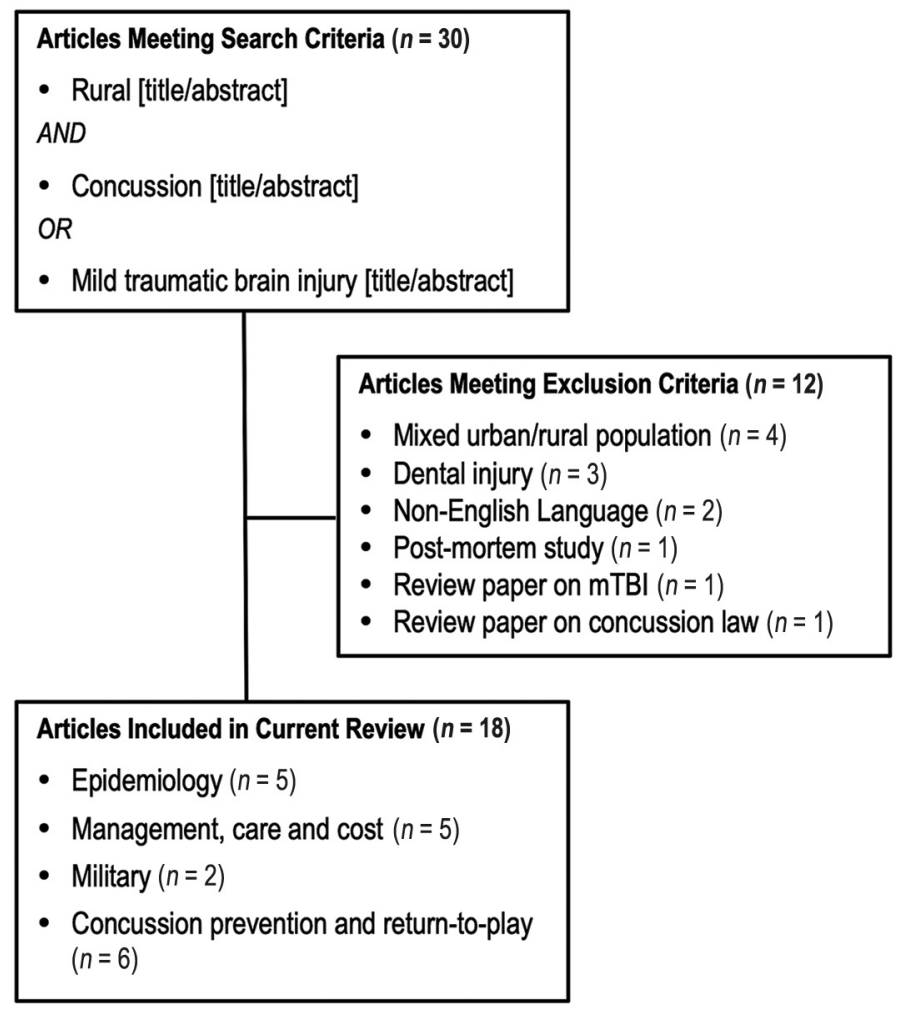

Fig. 1 Flow diagram of included articles.

rural settings regarding helmet and seatbelt use, as motorized vehicles are often used on farms. Conversely, urban children were five times more likely to be injured from pedestrian versus auto ( 24 vs. $5 \%$ ) due to high-population density, fast moving traffic, and absence of parks. ${ }^{25}$

Poland adult study: In 265 patients (34\% rural) with multiorgan injuries at a Polish regional hospital from 1999 to $2002,{ }^{26}$ Karwat et al reported that rural residence was a risk factor for MVAs with an injured driver, and for home-based injuries. Concussion was twice as frequent in urban (59.9\%) versus rural (31.1\%) inhabitants, while midbrain injuries were more likely in rural patients (46.7 vs. $24.6 \%) .{ }^{26}$ Approximately, $57 \%$ of urban and $50 \%$ of rural patients had polytrauma.

India mixed adult/pediatric study: Munivenkatappa et al reported on the epidemiology of bicycle-related TBIs at a tertiary referral center in India, over a period of 6 months in $2011 .{ }^{27}$ In 108 patients, 68.5\% were rural, 91.6\% had poor socioeconomic status, mean age was 27.7 years, and none wore a helmet. Forty-nine percent had head-computed tomography (CT) abnormalities (skull fracture: $23.0 \%$, contusion: $19.4 \%$, and extra-axial hemorrhage: 6.5-9.3\%). Laborers (versus students) had odds ratio $(\mathrm{OR})=9.1$ for severe TBI. No statistical differences were found between rural/urban settings for occupation, mechanism, injury severity, CT abnormalities, or mortality; however, $9.4 \%$ of rural patients died compared with $2.9 \%$ of urban patients. At a mean outcome time point of 4.5 months, $82 \%$ reported good functional recovery, $20 \%$ reported headaches, $18 \%$ fatigue, and $15 \%$ tiredness at work. The authors did not differentiate urban versus rural differences in outcome.
China epidemiological study: In a population-based study of neurotrauma in China using door-to-door surveys across six large cities and 21 rural and minority areas from 1983 to 1985, Zhao and Wang reported urban and rural incidences of 55.4/100,000 and 64.1/100,000, respectively. ${ }^{28}$ Mortality in urban areas was $6.3 / 100,000$, compared with $9.7 / 100,000$ in rural areas. ${ }^{28}$ The male:female ratio was $62.4 / 37.6 \%$ in urban and $71.3 / 28.7 \%$ in rural areas. Adult versus pediatric injuries were not specifically stated.

United States pediatric study: In a U.S. study of sports concussion-related hospitalizations aged 5 to 18 years old from 2000 to 2004 using the Nationwide Inpatient Sample (NIS), weighted annual nonfatal admissions for diagnosed concussions (International Classification of Diseases, 9th Revision [ICD-9]) were 928, which accounted for nearly half of all pediatric TBI admissions. ${ }^{29}$ Twelve percent were admitted to rural hospitals and $42 \%$ to nonteaching hospitals. Only $12 \%$ received neuroimaging studies. Rural $(O R=1.75)$ and nonteaching ( $O R=1.91)$ hospitals were more likely to admit concussions compared with nonconcussion TBI. ${ }^{29}$ The authors postulate that rural providers may be more conservative in management, coupled with lower access to MRI or other diagnostic tools. There may be a difference in level of experience or availability of consultants in rural settings. It remains debatable whether admission for observation is cost-effective, especially without neuroimaging. To date, guidelines on frequency of mTBI follow-up do not exist. Patients discharged to home remain at risk of sequelae, and may benefit from observation by informed caregivers and/or guardians, with strict return precautions. Caregivers may influence patient 
Table 1 Summary of included studies

\begin{tabular}{|c|c|c|c|c|c|c|}
\hline \multicolumn{7}{|c|}{ Epidemiology } \\
\hline $\begin{array}{l}\text { Author } \\
\text { (year) }\end{array}$ & Study type & $n$ & $\begin{array}{l}\text { Age and } \\
\text { sex }\end{array}$ & Description & $\begin{array}{l}\text { Outcome } \\
\text { measures }\end{array}$ & Results \\
\hline $\begin{array}{l}\text { Karwat et al } \\
\text { (2009) }\end{array}$ & $\begin{array}{l}\text { Head injuries, } \\
\text { retrospective } \\
\text { cohort study }\end{array}$ & $\begin{array}{l}265 \\
\text { head-in- } \\
\text { jured } \\
\text { patients }\end{array}$ & $\begin{array}{l}\text { 34\% from } \\
\text { rural set- } \\
\text { tings } \\
\text { Mean age: } \\
\text { urban } \\
46.4 \text { years } \\
\text { (SD }=18.7) \text {, } \\
\text { rural } 39.1 \\
\text { (SD = 17.9) } \\
\text { Urban } \\
\text { male/ } \\
\text { female: } \\
74.3 / 25.7 \% \\
\text { Rural: } \\
82.2 / 17.8 \%\end{array}$ & $\begin{array}{l}\text { Analysis of head } \\
\text { injuries treated at } \\
\text { a regional hospital } \\
\text { from 1999-2002 } \\
\text { with emphasis on } \\
\text { demographics and } \\
\text { health }\end{array}$ & $\begin{array}{l}\text { Patient demo- } \\
\text { graphics associa- } \\
\text { tions with area of } \\
\text { residence }\end{array}$ & $\begin{array}{l}\text { In rural areas, ages } 35-49 \text { years and } \\
\geq 65 \text { were the most common present- } \\
\text { ing populations, while ages } 20-34 \\
\text { were most common in urban areas. } \\
\text { Rural patients were significantly less } \\
\text { educated }(p<0.001) \text { and more likely } \\
\text { to be married }(p<0.05)\end{array}$ \\
\hline $\begin{array}{l}\text { Munivenkat- } \\
\text { appa et al27 } \\
(2013)\end{array}$ & $\begin{array}{l}\text { Bicycle head } \\
\text { injuries, } \\
\text { retrospective } \\
\text { cohort study }\end{array}$ & $\begin{array}{l}108 \\
\text { head-in- } \\
\text { jured } \\
\text { patients }\end{array}$ & $\begin{array}{l}\text { Mean age, } \\
27.8 \text { years } \\
\text { (range: } \\
2-76 \text { years) } \\
\text { Male/ } \\
\text { female: } \\
97 / 7 \%\end{array}$ & $\begin{array}{l}\text { Epidemiology of } \\
\text { head injuries due to } \\
\text { bicycle accidents in } \\
\text { India }\end{array}$ & \begin{tabular}{|l|} 
Area of residence, \\
etiology of injury, \\
socioeconomic \\
status (SES), place \\
of presentation, \\
clinical and imaging \\
findings, and mor- \\
tality
\end{tabular} & $\begin{array}{l}\text { From rural areas, } 68.5 \%, 91.6 \% \text { from } \\
\text { poor SES, } 55.6 \% \text { MVAs. } 62 \% \text { of rural } \\
\text { versus } 26 \% \text { of urban patients initially } \\
\text { presented to primary care. } 49 \% \text { had } \\
\text { abnormal CT, and } 7.4 \% \text { died due to } \\
\text { injuries. No significant differences } \\
\text { were found for occupation, mecha- } \\
\text { nism, injury severity, and CT findings } \\
\text { between rural and urban groups }\end{array}$ \\
\hline $\begin{array}{l}\text { Stewart } \\
\text { et } \mathrm{a}^{25}(2014)\end{array}$ & $\begin{array}{l}\text { Concussion, } \\
\text { retrospective } \\
\text { cohort study }\end{array}$ & \begin{tabular}{|l|}
2,112 \\
pediatric \\
concussion \\
patients
\end{tabular} & $\begin{array}{l}\text { Medi- } \\
\text { an age, } \\
13 \text { years } \\
(\text { IQR }=6) \\
\text { Male/ } \\
\text { female: } \\
67 / 33 \%\end{array}$ & $\begin{array}{l}\text { Characterization } \\
\text { and comparison of } \\
\text { pediatric concussion } \\
\text { incidence from } \\
\text { urban and rural } \\
\text { areas presenting } \\
\text { to a regional level-I } \\
\text { trauma center }\end{array}$ & $\begin{array}{l}\text { Concussion etiolo- } \\
\text { gy, rates and mech- } \\
\text { anism stratified } \\
\text { by age group and } \\
\text { urban versus rural } \\
\text { residence }\end{array}$ & $\begin{array}{l}\text { Rural had more MVC-related con- } \\
\text { cussions }(p<0.001) \text {, and were older, } \\
\text { than urban patients }(p=0.019) \text {. Rural } \\
\text { concussion rates were more than } \\
\text { twice that of urban }(300 / 1000 \text { versus } \\
140 / 1000) \text {. More rural concussions } \\
\text { with LOC }(p<0.001) \text { and intracra- } \\
\text { nial injuries }(p<0.001) \text {. More rural } \\
\text { patients were inpatient ( } p<0.001) \\
\text { and admitted to special care unit, } \\
\text { while urban patients were more likely } \\
\text { to be sent home directly from ED ( } p \\
=0.012)\end{array}$ \\
\hline $\begin{array}{l}\text { Yang et } a^{29} \\
(2008)\end{array}$ & $\begin{array}{l}\text { TBI/con- } \\
\text { cussion, } \\
\text { retrospective } \\
\text { cohort study }\end{array}$ & $\begin{array}{l}1,568 \mathrm{TBI} \\
(755 \text { from } \\
\text { concus- } \\
\text { sion) }\end{array}$ & $\begin{array}{l}\text { Age range } \\
5-18 \text { years } \\
\text { TBI: male/ } \\
\text { female } \\
82.8 / 17.2 \% \\
\text { Concus- } \\
\text { sion: male/ } \\
\text { female } \\
84 / 16 \%\end{array}$ & $\begin{array}{l}\text { Analysis of patient } \\
\text { and hospital charac- } \\
\text { teristics associated } \\
\text { with hospitalization } \\
\text { for non-fatal sports } \\
\text { concussions in } \\
\text { urban versus rural } \\
\text { hospitals }\end{array}$ & $\begin{array}{l}\text { Demographics, } \\
\text { insurance status, } \\
\text { LOS, number of } \\
\text { hospitalizations, } \\
\text { number of LOC } \\
\text { events, procedures } \\
\text { received }\end{array}$ & $\begin{array}{l}\text { Among all patients, } 52.3 \% \text { with } \\
\text { concussion had LOC, } 80 \% \text { received } \\
\text { no in-hospital procedures, mean } \\
\text { LOS } 1.1 \text { days, } 4.9 \% \text { of hospitalized } \\
\text { patients were uninsured. Teaching } \\
\text { hospitals (versus non-teaching) had } \\
\text { greater odds of admitting sports-re- } \\
\text { lated concussions (OR }=1.91 ; 95 \% \\
\mathrm{Cl} 1.44-2.53 \text { ). Rural hospitals with } \\
\text { greater odds of admitting sports-re- } \\
\text { lated concussions (OR }=1.75 ; 95 \% \mathrm{Cl} \\
1.11-2.77 \text { ) }\end{array}$ \\
\hline $\begin{array}{l}\text { Zhao et al }{ }^{28} \\
(2001)\end{array}$ & $\begin{array}{l}\text { TBI, descrip- } \\
\text { tive epidemi- } \\
\text { ology study }\end{array}$ & $\begin{array}{l}63,195 \\
\text { surveys }\end{array}$ & $\begin{array}{l}\text { Male/ } \\
\text { female: } \\
63.4 / 37.6 \%\end{array}$ & $\begin{array}{l}\text { Neurologist and } \\
\text { neurosurgeon-led, } \\
\text { population-based } \\
\text { door-to-door survey } \\
\text { on TBI in } 6 \text { major } \\
\text { cities and } 21 \text { rural/ } \\
\text { minority areas in } \\
\text { People's Republic of } \\
\text { China }\end{array}$ & \begin{tabular}{|l|} 
History of TBI with \\
coma or retrograde \\
amnesia, residual \\
deficits or abnormal \\
imaging
\end{tabular} & $\begin{array}{l}\text { Overall incidence of TBI was } \\
55.4 / 100,000(64.1 / 100,000) \text { in } \\
\text { rural/minority areas. The majority } \\
\text { were males }(71.3 \%) \text { in rural areas. } \\
\text { Mortality rate lower in urban versus } \\
\text { rural areas }(6.3 / 100,000 \text { versus } \\
9.7 / 100,000) \text { In all areas, concussion } \\
\text { was the most common injury }(68.4 \%)\end{array}$ \\
\hline
\end{tabular}


Table 1 (continued)

\begin{tabular}{|c|c|c|c|c|c|c|}
\hline $\begin{array}{l}\text { Author } \\
\text { (year) }\end{array}$ & Study type & $n$ & $\begin{array}{l}\text { Age and } \\
\text { sex }\end{array}$ & Description & $\begin{array}{l}\text { Outcome } \\
\text { measures }\end{array}$ & Results \\
\hline \multicolumn{7}{|c|}{ Management, care, and cost } \\
\hline $\begin{array}{l}\text { Brown et } \mathrm{al}^{31} \\
(2018)\end{array}$ & $\begin{array}{l}\text { mTBI, ret- } \\
\text { rospective } \\
\text { cohort study }\end{array}$ & $\begin{array}{l}540 \text { mTBI } \\
\text { patients }\end{array}$ & $\begin{array}{l}\text { All } \\
\geq 16 \text { years } \\
\text { old; } 73.6 \% \\
<55 \text { years } \\
\text { Male/ } \\
\text { female: } \\
66.2 / 37.8 \%\end{array}$ & \begin{tabular}{l|} 
12-month retro- \\
spective assessment \\
adherence to clinical \\
practice guidelines \\
(CPGs) at a rural/ \\
regional hospital
\end{tabular} & $\begin{array}{l}\text { Adherence to CPG } \\
\text { was measured by } \\
\text { ED mTBI/concus- } \\
\text { sion evaluation } \\
\text { criteria (4hrs } \\
\text { observation, criteria } \\
\text { met for CT, referrals } \\
\text { given, education } \\
\text { provided) }\end{array}$ & $\begin{array}{l}\text { Men aged 16-24 had a higher } \\
\text { proportion of mTBI }(p=0.013) \text { and } \\
\text { had more mTBIs due to collisions ( } p \\
<0.01) \text {. Older patients more likely } \\
\text { to receive CT }(p=0.009) \text {. Of those } \\
\text { meeting CT criteria }(N=245) \text {, only } \\
65.3 \% \text { received a CT scan, } 45 \% \text { had } \\
\text { 4-hour observation, and } 6.3 \% \text { were } \\
\text { appropriately directed to referral ser- } \\
\text { vices. Only } 74.1 \% \text { received safe dis- } \\
\text { charge and } 33 \% \text { received education }\end{array}$ \\
\hline $\begin{array}{l}\text { Graves } \\
\text { et a }{ }^{34} \\
(2018)\end{array}$ & $\begin{array}{l}\text { mTBI, ret- } \\
\text { rospective } \\
\text { cohort study }\end{array}$ & $\begin{array}{l}387,846 \\
\text { pediat- } \\
\text { ric TBI } \\
\text { patients }\end{array}$ & $\begin{array}{l}\text { Urban: } \\
\text { mean age } \\
8.7 \text { years, } \\
\text { rural: } \\
10.0 \text { years } \\
\text { Urban } \\
\text { male/ } \\
\text { female: } \\
61.6 / 38.4 \%, \\
\text { rural } \\
62 / 38 \%\end{array}$ & $\begin{array}{l}\text { 180-day analy- } \\
\text { ses of healthcare } \\
\text { utilization and cost } \\
\text { in urban and rural } \\
\text { pediatric patients } \\
\text { after mTBI }\end{array}$ & $\begin{array}{l}\text { Residing location, } \\
\text { healthcare utiliza- } \\
\text { tion, and healthcare } \\
\text { costs }\end{array}$ & $\begin{array}{l}\text { Rural patients comprised a minor- } \\
\text { ity of all patients (13\%). Rural less } \\
\text { likely to have speech therapy, mental } \\
\text { health, and other outpatient services. } \\
\text { Overall healthcare costs were } 11 \% \\
\text { higher in rural compared with urban } \\
\text { children (adjusted CR: } 1.11,95 \% \mathrm{Cl} \text { : } \\
1.06-1.16)\end{array}$ \\
\hline $\begin{array}{l}\text { Levy et al }{ }^{32} \\
(2013)\end{array}$ & $\begin{array}{l}\text { mTBI with } \\
\text { small ICH, } \\
\text { retrospective } \\
\text { cohort study }\end{array}$ & $\begin{array}{l}76 \text { with } \\
\text { mTBI and } \\
\text { small ICH, } \\
\text { without } \\
\text { coagulop- } \\
\text { athy }\end{array}$ & $\begin{array}{l}71 \% \\
<55 \text { years } \\
\text { Male/ } \\
\text { female: } \\
70 / 30 \%\end{array}$ & $\begin{array}{l}\text { 6-year assessment } \\
\text { of a non-transfer } \\
\text { protocol to a Level } \\
\text { I Trauma Center } \\
\text { from a rural Level } \\
\text { III Trauma Center } \\
\text { without neurosurgi- } \\
\text { cal service }\end{array}$ & $\begin{array}{l}\text { LOS and neurosur- } \\
\text { gical intervention } \\
\text { rate }\end{array}$ & $\begin{array}{l}\text { All patient CTs were assess by Level I } \\
\text { trauma center neurosurgeon before } \\
\text { deciding nonstransfer. Median LOS } \\
\text { was } 1 \text { day. None of the patients } \\
\text { required postadmission transfer or } \\
\text { neurosurgical intervention }\end{array}$ \\
\hline $\begin{array}{l}\text { Schoot- } \\
\text { man and } \\
\text { Fuortes }{ }^{33} \\
(2000)\end{array}$ & $\begin{array}{l}\text { mTBI, descrip- } \\
\text { tive epidemi- } \\
\text { ology study }\end{array}$ & $\begin{array}{l}1.4 \text { million } \\
\text { visits for } \\
\text { TBI }\end{array}$ & $N / A$ & $\begin{array}{l}\text { Retrospective } \\
\text { database analysis of } \\
\text { patients presenting } \\
\text { with TBI to U.S. } \\
\text { physician offices, } \\
\text { outpatient centers, } \\
\text { and EDs from } \\
\text { 1995-1997 } \\
\end{array}$ & $\begin{array}{l}\text { Annual TBI inci- } \\
\text { dence, differences } \\
\text { between urban and } \\
\text { rural areas }\end{array}$ & $\begin{array}{l}\text { Annual rate of mTBI related medical } \\
\text { visits was } 5.7 / 1,000 \text { in rural versus } \\
4.1 / 1,000 \text { in urban populations. More } \\
\text { rural patients were transferred to } \\
\text { outside facilities as compared with } \\
\text { urban patients ( } 8.5 \text { vs. } 2.7 \% \text { ) }\end{array}$ \\
\hline $\begin{array}{l}\text { Vargas } \\
\text { et al }{ }^{36} \\
(2012)\end{array}$ & $\begin{array}{l}\text { mTBI, case } \\
\text { report }\end{array}$ & $\begin{array}{l}1 \text { patient } \\
\text { after } \mathrm{mTBI} \\
\text { with } \mathrm{LOC}\end{array}$ & $\begin{array}{l}\text { 15-year old } \\
\text { male }\end{array}$ & \begin{tabular}{|l|} 
Telemedicine \\
consult to evaluate \\
postconcussive \\
symptoms 19-days \\
after mTBI. Patient \\
from rural area ser- \\
viced by a hospital \\
without neurological \\
service
\end{tabular} & $\begin{array}{l}\text { Neurological exam } \\
\text { and NCT using } \\
\text { SCAT2, HIT-6, GAD } \\
\text { 7-item, and PHQ-9 }\end{array}$ & $\begin{array}{l}\text { Prior to telemedicine consultation, } \\
\text { patient was assessed as able to } \\
\text { return to normal sports play. Upon } \\
\text { teleconcussion consultation, patient } \\
\text { was determined to be symptomatic } \\
\text { with moderate balance impairments } \\
\text { and referred for formal face-to-face } \\
\text { evaluation with neurology provider }\end{array}$ \\
\hline \multicolumn{7}{|c|}{ Military and postdeployment } \\
\hline $\begin{array}{l}\text { Tsai et al }{ }^{37} \\
(2012)\end{array}$ & $\begin{array}{l}\text { Concussion, } \\
\text { retrospective } \\
\text { cohort study }\end{array}$ & $\begin{array}{l}233 \text { veter- } \\
\text { ans }\end{array}$ & $\begin{array}{l}\text { Mean age } \\
35.9 \text { years } \\
(\mathrm{SD}=9.9) \\
\text { Male/ } \\
\text { female: } \\
92 / 8 \%\end{array}$ & $\begin{array}{l}\text { Survey of com- } \\
\text { bat-related con- } \\
\text { cussion and PTSD } \\
\text { sequelae from VA } \\
\text { system in in rural } \\
\text { and urban settings } \\
\text { in Hawaii }\end{array}$ & $\begin{array}{l}\text { Concussion, } \\
\text { postconcussive } \\
\text { symptoms, and } \\
\text { PTSD screening; } \\
\text { and HRQOL }\end{array}$ & $\begin{array}{l}\text { Patients with positive screens for } \\
\text { concussion and postconcussive } \\
\text { symptoms were more likely to have } \\
\text { PTSD }(p<0.001) \text {, alcohol use issues } \\
(p>0.01) \text {, and live in rural areas ( } p \\
<0.01) \text {. These patients were also like- } \\
\text { lier to score lower on health-related } \\
\text { quality of life measures }(p<0.001)\end{array}$ \\
\hline
\end{tabular}


Table 1 (continued)

\begin{tabular}{|c|c|c|c|c|c|c|}
\hline \multicolumn{7}{|c|}{ Epidemiology } \\
\hline $\begin{array}{l}\text { Author } \\
\text { (year) }\end{array}$ & Study type & $n$ & $\begin{array}{l}\text { Age and } \\
\text { sex }\end{array}$ & Description & $\begin{array}{l}\text { Outcome } \\
\text { measures }\end{array}$ & Results \\
\hline \multicolumn{7}{|c|}{ Concussion prevention and return to play } \\
\hline $\begin{array}{l}\text { Chrisman } \\
\text { et } \mathrm{al}^{45} \\
(2014)\end{array}$ & $\begin{array}{l}\text { mTBI, descrip- } \\
\text { tive epidemi- } \\
\text { ology study }\end{array}$ & $\begin{array}{l}270 \text { coach- } \\
\text { es, ath- } \\
\text { letes, and } \\
\text { parents }\end{array}$ & $\begin{array}{l}72.1 \% \\
<51 \text { years }\end{array}$ & $\begin{array}{l}\text { Survey of education } \\
\text { level 3-years after } \\
\text { implementation of } \\
\text { concussion legisla- } \\
\text { tion in high school } \\
\text { sports programs in } \\
\text { Washington state } \\
\text { (United States). } \\
\text { Knowledge levels } \\
\text { of coaches, parents } \\
\text { and athletes residing } \\
\text { in urban or rural are- } \\
\text { as were compared }\end{array}$ & $\begin{array}{l}\text { Mixed methods } \\
\text { survey (online and } \\
\text { paper) and inter- } \\
\text { views of coaches re- } \\
\text { garding concussion } \\
\text { education and eval- } \\
\text { uation modalities }\end{array}$ & $\begin{array}{l}\text { Among coaches, } 74.4 \% \text { were required } \\
\text { concussion education prior to receiv- } \\
\text { ing coaching privileges, although } \\
16.9 \% \text { were allowed to coach regard- } \\
\text { less of education status. } 93.9 \% \text { of } \\
\text { coaches were mandated to complete } \\
\text { training annully. } 91 \% \text { of coaches } \\
\text { received education by } \geq 2 \text { modalities } \\
\text { (paper, video, presentation, test, in- } \\
\text { person). Coaches reported that } 89.3 \\
\text { and } 82.9 \% \text { of athletes and parents } \\
\text { were educated on concussion. Urban } \\
\text { coaches were more likely to have } \\
\text { athletic trainers (ATs; } p<0.001 \text { ) and } \\
\text { physician attendance at least half } \\
\text { of games }(p=0.03) \text {. Urban coaches } \\
\text { more likely to report athletes with } \\
\text { concussions }(p<0.001)\end{array}$ \\
\hline $\begin{array}{l}\text { Kroshus } \\
\text { et al }{ }^{42} \\
(2017)\end{array}$ & $\begin{array}{l}\text { Concussion, } \\
\text { cross-sectional } \\
\text { study }\end{array}$ & \begin{tabular}{|l|}
270 teams, \\
144 \\
schools
\end{tabular} & $N / A$ & $\begin{array}{l}\text { Survey of diagnosed } \\
\text { concussions at } \\
\text { high schools with } \\
\text { or without ATs on } \\
\text { staff. Schools further } \\
\text { stratified by number } \\
\text { of students enrolled } \\
\text { in subsidized school } \\
\text { lunch programs }\end{array}$ & $\begin{array}{l}\text { Number of concus- } \\
\text { sions in previous } \\
\text { season, school loca- } \\
\text { tion (urban versus } \\
\text { rural), employment } \\
\text { of AT, \% of students } \\
\text { eligible for subsi- } \\
\text { dized school lunch }\end{array}$ & $\begin{array}{l}\text { Among athletes, } 74.6 \% \text { suffered } \\
\text { concussions, } 34.7 \% \text { of schools were } \\
\text { in rural locations, } 70.8 \% \text { of schools } \\
\text { employed ATs, and } 42.8 \% \text { of students } \\
\text { qualified for subsidized school lunch- } \\
\text { es. ATs were significantly more likely } \\
\text { to be employed in urban regions ( } p \\
<0.001) \text { and at schools that had less } \\
\text { children eligible for subsidized school } \\
\text { lunch }(p=0.005)\end{array}$ \\
\hline $\begin{array}{l}\text { Lyons et al }{ }^{47} \\
(2017)\end{array}$ & $\begin{array}{l}\text { Concussion, } \\
\text { descriptive } \\
\text { epidemiology } \\
\text { study }\end{array}$ & \begin{tabular}{|l|}
151 re- \\
spondents
\end{tabular} & $\begin{array}{l}\text { Mixed } \\
\text { members } \\
\text { of a school } \\
\text { communi- } \\
\text { ty: admin- } \\
\text { istrators, } \\
\text { teachers, } \\
\text { school } \\
\text { nurses }\end{array}$ & $\begin{array}{l}\text { Stakeholders in rural } \\
\text { and urban schools } \\
\text { were surveyed } \\
\text { to understand } \\
\text { disparities in lack } \\
\text { of Return to Learn } \\
\text { (RTL) Guidelines } \\
\text { following concussion } \\
\text { in children }\end{array}$ & $\begin{array}{l}\text { Survey responses } \\
\text { on unmet need } \\
\text { domains and } \\
\text { identification of } \\
\text { recommendations }\end{array}$ & $\begin{array}{l}\text { Unmet needs surrounding concus- } \\
\text { sion treatment and RTL Guidelines } \\
\text { included: lack of school policies, } \\
\text { barriers to providing or receiving } \\
\text { accommodations, wide variability in } \\
\text { communication patterns, and need } \\
\text { to develop a formal school RTL policy } \\
\text { for improved training and adoption }\end{array}$ \\
\hline $\begin{array}{l}\text { McGrath } \\
\text { et al }{ }^{43} \\
(2018)\end{array}$ & $\begin{array}{l}\text { Concussion, } \\
\text { focus group }\end{array}$ & $\begin{array}{l}\text { Focus } \\
\text { group of } \\
\text { five stake- } \\
\text { holders in } \\
\text { Nebraska } \\
\text { state } \\
\text { (United } \\
\text { States) }\end{array}$ & \begin{tabular}{|l} 
(Four \\
certified \\
ATs: one at \\
high school, \\
one at \\
university, \\
one sports \\
medicine \\
director, \\
one outpa- \\
tient clinic \\
director; \\
and one \\
medical \\
software \\
engineer) \\
\end{tabular} & $\begin{array}{l}\text { Design of a mobile } \\
\text { medical application } \\
\text { that connects high } \\
\text { school athletes } \\
\text { and coaches with } \\
\text { ATs for concussion } \\
\text { assessment }\end{array}$ & $\begin{array}{l}\text { Local maps of } \\
\text { Nebraska were } \\
\text { assessed for con- } \\
\text { centration of ATs } \\
\text { employed at high } \\
\text { schools }\end{array}$ & $\begin{array}{l}\text { Rural areas were in greatest need of } \\
\text { ATs and would be the target audience } \\
\text { for medical app. The focus group } \\
\text { defined a mobile application that } \\
\text { valued enhancing quality of care, } \\
\text { efficiency, and extending scope of } \\
\text { care as most significant. Application } \\
\text { will implement the Sport Concussion } \\
\text { Assessment Tool } 3 \text { (SCAT3), provide } \\
\text { information and real-time feedback } \\
\text { to ATs remotely for evaluation }\end{array}$ \\
\hline $\begin{array}{l}\text { Murphy } \\
\text { et al }{ }^{44} \\
(2012)\end{array}$ & $\begin{array}{l}\text { mTBI, } \\
\text { cross-sectional } \\
\text { study }\end{array}$ & $\begin{array}{l}48 \text { with } \\
\text { mTBI }\end{array}$ & $N / A$ & $\begin{array}{l}\text { Evaluated the } \\
\text { practices of football } \\
\text { coaches and ATs on } \\
\text { concussion manage- } \\
\text { ment of high school } \\
\text { athletes in urban } \\
\text { versus rural school } \\
\text { districts }\end{array}$ & \begin{tabular}{|l|} 
Differences in \\
management \\
practices between \\
rural versus urban \\
school districts and \\
between coaches \\
and ATs in use of \\
SCAT2 and NCT
\end{tabular} & $\begin{array}{l}\text { Among respondents, } 56 \% \text { used } \\
\text { SCAT2 for on-field assessment; urban } \\
\text { respondents significantly more likely } \\
\text { to use SCAT2 ( } 68 \text { vs. } 35 \%, p=0.015) \text {. } \\
31 \% \text { used NCT, significantly more } \\
\text { common in urban settings ( } 59 \text { vs. } \\
4 \%, p<0.01 \text { ). ATs more likely to use } \\
\text { standardized assessment instruments }\end{array}$ \\
\hline
\end{tabular}


Table 1 (continued)

\begin{tabular}{|c|c|c|c|c|c|c|}
\hline \multicolumn{7}{|c|}{ Epidemiology } \\
\hline $\begin{array}{l}\text { Author } \\
\text { (year) }\end{array}$ & Study type & $n$ & $\begin{array}{l}\text { Age and } \\
\text { sex }\end{array}$ & Description & $\begin{array}{l}\text { Outcome } \\
\text { measures }\end{array}$ & Results \\
\hline $\begin{array}{l}\text { Rivara et al }{ }^{45} \\
\text { (2014) }\end{array}$ & $\begin{array}{l}\text { Concussion, } \\
\text { prospective } \\
\text { cohort study }\end{array}$ & $\begin{array}{l}778 \text { dyads } \\
\text { of student } \\
\text { athletes } \\
\text { and par- } \\
\text { ents }\end{array}$ & $\begin{array}{l}\text { Age } 14- \\
19 \text { years } \\
\text { Male/ } \\
\text { female: } \\
67 / 37 \% \\
\text { female }\end{array}$ & $\begin{array}{l}\text { Survey of sports } \\
\text { concussions in high } \\
\text { school athletes } \\
\text { and percentage of } \\
\text { athletes playing with } \\
\text { concussive symp- } \\
\text { toms during Fall } \\
2012 \text { sports season. } \\
\text { Evaluated effect of } \\
\text { coach education } \\
\text { and likelihood of } \\
\text { an athlete playing } \\
\text { with concussive } \\
\text { symptoms }\end{array}$ & $\begin{array}{l}\text { Rate of concussion } \\
\text { per } 1,000 \text { athletes, } \\
\% \text { of students play- } \\
\text { ing with concussion } \\
\text { symptoms, \% of } \\
\text { concussed athletes } \\
\text { stating coach was } \\
\text { aware of concus- } \\
\text { sion }\end{array}$ & $\begin{array}{l}\text { Concussion incidence } 3.6 / 1,000 \\
\text { athletes, } 69 \% \text { of concussed athletes } \\
\text { played with symptoms, and } 40 \% \\
\text { reported that the coach was not } \\
\text { aware. Coaches trained by video and } \\
\text { quiz were } 50 \% \text { and } 40 \% \text { less likely } \\
\text { to be aware of concussions. Rate of } \\
\text { concussion for football players was } \\
6.3 \text { times higher during games versus } \\
\text { practices. Rate of concussion for } \\
\text { soccer in girls was } 12.4 \text { times higher } \\
\text { during games versus practices }\end{array}$ \\
\hline
\end{tabular}

Abbreviations: AT, athletic trainer; $\mathrm{Cl}$, confidence interval; $\mathrm{CR}$, cost ratio; $\mathrm{CT}$, computed tomography; $\mathrm{ED}$, emergency department; IQR, interquartile range; GAD, generalized anxiety disorder; HIT-6, Headache Impact Test-6; HRQOL, health-related quality of life; ICH, intracerebral hemorrhage; LOC, loss of consciousness LOS, length of stay; mTBI, mild-traumatic brain injury; N/A, not available; NCT, neurocognitive testing; PTSD, posttraumatic stress disorder; RTL, return to learn; RTP, return to play; SCAT2, Standardized Concussion Assessment Tool, Second Edition; SD, standard deviation; SES, socioeconomic status; VA, veterans affairs.

trajectory of recovery, dependent upon socioeconomic status, caregiver education, and stress level. ${ }^{30}$

\section{Management, Care, and Cost}

EDs are often the only point of health care contact for mTBI/ concussion patients in rural settings. ${ }^{31}$ While clinical practice guidelines (CPGs), for example, neuroimaging, observation, safe discharge, and TBI education, exist for ED management, adherence remains underinvestigated in rural areas. Brown et al reviewed records from 1,280 mTBI patients at a regional Australian ED and found (1) 65\% of those qualifying for CT actually received a scan, (2) underrecording of 4-hour observation rates (45\%) and safe discharges (74\%), (3) only 33\% received $\mathrm{mTBI}$ education, and (4) only $6 \%$ of patients who received a CT were referred to clinic. ${ }^{31}$ Adherence to CPGs was likely suboptimal, and referral services were underutilized in certain regional centers.

Historically, there is a reluctance for rural hospitals without neurosurgical capabilities to admit patients with positive head CTs due to concern for sudden deterioration. Levy et al evaluated a novel nontransfer protocol in 76 noncoagulopathic mTBI patients with CT evidence of small intracranial hemorrhage (e.g., small subarachnoid hemorrhage, punctate/minimal contusion or intraparenchymal hemorrhage, and thin subdural hemorrhage without mass effect) at a rural level-III trauma center without neurosurgical capabilities from 2007 to $2012 .{ }^{32}$ CT scans were reviewed by an on-call neurosurgeon at a level-I trauma center and consensus was obtained for nontransfer. Patients were managed by the trauma service with hourly neurological assessments. No in-hospital deaths occurred and all patients were discharged with stable head CT and in good neurologic condition. ${ }^{32}$ The authors report the potential of health care resource/cost conservation with proper implementation of nontransfer protocols.
Schootman and Fuortes reported 1.4 million annual incidence of mTBI-related ambulatory care visits from 1995 to 1997 in a national study of all nonfederal, nonhospital-based office or clinic visits. ${ }^{33}$ The rate of visits to rural facilities were lower than urban $(4.1 / 1,000$ vs. 5.7/1,000). Hospitalization rate was similar at $15.0 \%$ for rural and $18.2 \%$ for urban. Higher proportion of rural patients were transferred to a different facility ( 8.5 vs. $2.7 \%$ ). The authors postulate that urban injuries were more likely to be severe and require higher level of care. ${ }^{33}$ It should not be neglected that while hospitalization rates were the same, rates of transfer to different facility was substantially higher in rural communities, underscoring the challenge a mTBI patient may face in the rural setting in seeking definitive ambulatory evaluation.

Regarding cost of pediatric concussions, annual charges documented by the NIS were 6 million USD per year from 2005 to $2014 .^{29}$ In a recent study using a commercial claims database, Graves et al evaluated 387,846 pediatric mTBI patients (13\% rural). ${ }^{34}$ Rural children were older, with higher incidence of polytrauma (51 vs. 43\%). Costs within 180 days after mTBI in rural children were 2,778 USD (vs. urban 2,499 USD), with an adjusted cost ratio of 1.11 . Rural children were less likely to work with speech therapy (incidence risk ratio $[I R R]=0.52)$, psychiatry/psychology $(I R R=0.63)$, and other outpatient services (IRR $=0.96$ ), however, were more likely to have physical therapy/occupational therapy (PT/OT) $($ IRR = 1.15). The authors attribute decreased utilization of psychiatry/psychology services to possible mental health stigma in rural communities and limitations in logging specialist versus generalist services, while the increased PT/OT utilization may stem from more severe injuries in rural patients. ${ }^{34}$ The article discusses the importance of sustained follow-up and offers telemedicine as an alternative to patients with distance barriers. 
Telemedicine is an emerging method of diagnosis and triage that enables immediate assessment without incurring otherwise mandatory transfer costs. ${ }^{35}$ Nearly one-third of the Arizona state (United States) population live in rural communities with limited access to specialized medical care. Vargas et al present the case of a 15 -year-old male athlete who sustained a concussion while playing soccer with Glasgow Coma Scale (GCW) 8 upon local ED arrival, which normalized over 4 hours. ${ }^{36}$ He was discharged after 24 hours of observation, however, continued to have postconcussive symptoms. He was the first concussed athlete to be evaluated by a concussion specialist via telemedicine, completing a comprehensive neurologic exam and neurocognitive battery at 19 days. ${ }^{36}$ The authors describe telemedicine assessment of concussion ("teleconcussion") as a solution to bridge specialty care to underserved communities, reduce urban versus rural disparities in trauma care, and can be utilized across longitudinal care settings to assist in RTP evaluations.

\section{Military and Postdeployment Considerations}

Special considerations exist for military personnel deployed to the post-2000 Iraq and Afghanistan wars (Operation Enduring Freedom [OEF], Operation Iraqi Freedom [OIF], Operation New Dawn [OND]) who return to home to rural settings. Tsai et al reported on postconcussive symptoms and posttraumatic stress disorder (PTSD) in 233 deployed veterans using the Hawaii Veterans Affairs (VA) Registry. ${ }^{37}$ The authors found that patients who screened positive for combat-related concussion and postconcussive symptoms were more likely to live in a rural area (63 vs. $44 \%$ for those not screening positive). In a similar population of 116 rural and 117 urban veterans who served in OEF/OIF/OND, Whealin et al found rural veterans were less educated (high school or less: 30 vs. $12 \%$, college degree: 17 vs. $30 \%$ ), more likely to screen positive for PTSD ( $43 \mathrm{vs.} 30 \%$ ), have postconcussive ( 41 vs. $24 \%$ ), and alcohol use problems ( 20 vs. $8 \%$ ). ${ }^{38}$ Utilization of VA services including medications and mental health were comparable between rural/urban groups $(40 \% / 32 \%)$. The authors contend that economic and community resources can buffer the impact of stress-related disorders. In OEF/OIF/OND veterans, morbidity rates have been reported at $60 \%$ higher rates than from soldiers in cities and suburbs. ${ }^{38}$

\section{Concussion Prevention and Return to Play}

Over the past decade, with improved recognition of concussions as causes of morbidity and barriers of not only RTP but also long-term health, there is an increasing focus on injury prevention, identification, and management in youth sports in U.S. secondary schools. Athletes who continue to play through a concussion are at risk for potentially catastrophic neurologic sequelae, in addition to prolonging symptomatology if undiagnosed or allowed to RTP prematurely. Athletic trainers (ATs) are certified in injury prevention, evaluation, immediate care, and rehabilitation, ${ }^{39}$ and the American Academy of Pediatrics has stated that football teams should have ATs on sidelines of organized play. ${ }^{40}$ However, studies have found $70 \%$ of secondary schools have an AT on staff, and only
$37 \%$ have an AT in full-time capacity. ${ }^{41}$ Rural locations were identified as barriers to ATs in part due to socioeconomic status (SES) disadvantages and requiring further travel. Rural settings also likely have fewer available medical centers for evaluation should the need arise.

Kroshus et al conducted a study of 144 secondary schools in Washington state (United States) and found dramatically reduced rural AT availability compared with urban (38 vs. $88 \%$ ). ${ }^{42}$ Football teams at AT-present schools had significantly greater relative risk (RR) of providing a concussion diagnosis ( $2-5$ vs. $0-1$ concussions: $R R=1.7 ;>5$ vs. $0-1$ concussions: $R R=4.2) .{ }^{42}$ The study showed that rurality, low-enrollment schools, and lower SES were barriers to AT hiring. The substantial overlap among these three risk factors in the rural setting deserve further study and resource allocation, especially considering the deleterious effect of youth concussion on later QOL, and the health care system. There are efforts to develop mobile applications for telemedicine evaluation with focused, web-based clinical information for implementation in rural areas. ${ }^{43}$

Murphy et al evaluated awareness of concussion assessment methods across Washington State high school football coaches and ATs in urban versus rural school districts. ${ }^{44} \mathrm{~A}$ 12-item questionnaire was distributed by e-mail regarding concussion assessment methods on the field and on follow-up testing, and awareness of the state law mandating concussion evaluation and health care clearance after concussion, before RTP. Across 58 coaches/ATs, 56\% used the Standardized Concussion Assessment Tool 2 (SCAT2; urban $=68 \%$, rural $=35 \%$ ), and $31 \%$ used neurocognitive testing (urban $=59 \%$, rural $=$ $4 \%)$. All coaches/trainers reported familiarity with concussion law. Interestingly, Rivara et al evaluated the incidence of sports concussions and athletes who play with symptoms in 778 urban and rural high school athletes, and found that 10 to $11 \%$ of suffered concussions over the season, $69 \%$ reported playing with symptoms, and $40 \%$ reported that coach was not aware of symptoms. ${ }^{45}$ Chrisman et al surveyed coaches, athletes, and parents 3 years after passage of the Washington state concussion law. ${ }^{46}$ Nearly all coaches received $\geq 2$ modalities of concussion education, while $37 \%$ of athletes and $16 \%$ of parents received the same, without differences by urban/ rural locales. Hence, legislation alone may not be sufficient in reducing concussions, and policies will need to incorporate modifications in both education and cultural attitudes for coaches and athletes. Furthermore, clinicians should be aware that a significant subpopulation of athletes and parents may not be receiving concussion education.

While RTP laws exist in certain U.S. states, return-to-learn (RTL) services, that is, mandating clinical assessment before returning to school, constitute a relatively new concept. Lyons et al report on RTL services in Washington state from a survey of community members from 144 rural and urban schools, and identified our unmet needs as follows: (1) current lack of school policies, (2) barriers to providing/receiving accommodations, (3) variability in communication patterns, and (4) recommendations shared by all stakeholders. ${ }^{47} \mathrm{~A}$ checklist was developed consisting of readiness, implementation, and evaluation stages of RTL implementation for public schools statewide. 


\section{Discussion}

Rural populations face specific challenges in the setting of $\mathrm{mTBI} /$ concussion. Our review focuses on the epidemiology of and management guidelines for populations at high risk of sustaining an mTBI including veterans and athletes. Our goal is to synthesize various clinical associations to aid in the creation of future best practices in acute, ambulatory, and preventative care.

\section{International Epidemiology of Rural mTBI/Concussion} $\mathrm{mTBI} /$ concussion has a high-incidence worldwide. We must consider that differences among countries in rural versus urban city planning resources may impact public safety as it relates to TBI. For example, the lack of dedicated bike lanes and low helmet use in India likely contributes to the fact that rural patients have a five-fold increase in mortality compared with their urban counterparts in that country. However, across studies, rural residence was a significant risk factor for increased incidence of injury notably from motor vehicle accidents. As transport times are likely protracted due to reduced resource and medical setting availabilities, primary prevention, such as helmet and seatbelt use, and recognition of early signs and symptoms of concussion are paramount in rural settings. Reduced neuroimaging technology in rural hospitals often leads either to patient admission for observation or transfer to a higher level of care. This practice can place strain on small health care centers and/or incur excess cost to patients. Reports discuss the option of observation from family or caregivers in an effort to reduce health care costs and unnecessary admissions, which further underscores the need for more consistent emergency medicine triage and appropriate training of personnel to deliver high-quality $\mathrm{mTBI} /$ concussion education to patient and caregivers. $\mathrm{mTBI} /$ concussion guidelines for neuroimaging and admission exist, ${ }^{48-50}$ and an investigation into the extent of utilization in rural settings constitute an important future direction. Impact of differences in rural versus urban city planning between countries should also be noted, such as low-helmet use and lack of dedicated bike lanes in India, where rural patients had a five-fold increase in mortality rate.

\section{Rural Populations Have Limited Resources for Management and Care of mTBI/Concussion, and Carry a Higher Burden of Health Care Costs}

From triage, observation and diagnosis to ambulatory follow-up, rural mTBI/concussion patients received fewer resources compared with urban counterparts. In pediatric cases, rural patients underutilized rehabilitation resources (i.e., speech, PT/OT) but still incurred greater health care costs, ${ }^{34}$ underscoring unmet clinical needs. It has been shown that few farm workers have insurance or seek government benefits ${ }^{51}$ further increasing financial burden. In addition to $\mathrm{mTBI}-/$ concussion-specific education, concerted efforts to disseminate infrastructural resources to patients in rural settings have potential in improving secondary and tertiary injury prevention. Telemedicine can help address these disparities by increasing access to specialist care across longer travel distances without incurring extra costs to the patient. Levy et al highlights the utility of a telemedicine consultation with an on-call neurosurgeon to safely risk-stratify mTBI patients with positive head CTs allowing for either transfer to a higher level of care or local management. ${ }^{32}$ Immediate access to expert assessment can reduce burden to both health care systems and patients while optimizing outcomes. While telemedicine and the best practices can improve care and decrease cost, health care and community programs are needed in rural settings to reduce stigma toward seeking rehabilitation services, especially those of mental health.

\section{Rural Veterans Have Higher Morbidity following mTBI/ Concussion}

Veterans have disproportionately high rates of mTBI/concussion and postconcussive symptoms. Veterans with postconcussive symptoms, PTSD, or a history of $\mathrm{mTBI} /$ concussion are also more likely to live in rural areas after return from deployment. ${ }^{37,38}$ This presents a clear challenge for access to health care resources. Although reported rates of VA service utilization were comparable between rural and urban groups, discordantly higher morbidity rates highlight a need for increased rural access to community resources ${ }^{38}$ and to prevent further decline using maladaptive coping mechanisms, such as alcohol. ${ }^{19,21,38}$ Importantly, the services required are "low-tech," primarily encompassing mental health, and community resources. For this reason, targeted interventions aimed at reintegration and patient education are of particular importance.

\section{Rural Athletes Are Less Likely to be Evaluated Appropriately after mTBI/Concussion}

Rurality not only limits access to neurosurgical or neurological triage, but also increases the risk for $\mathrm{mTBI} /$ concussions in secondary school contact sports. Across all studies in this review, rural location was associated with increased athlete risk through the lack of $\mathrm{mTBI} /$ concussion education for athletes and caregivers, lack of appropriately trained athletic personnel on-site due to funding and availability, and decreased access to medical centers for prompt evaluation. Although, where applicable, coaches across rural and urban settings were educated in RTP laws, only $56 \%$ used validated concussion assessment tools, such as the SCAT2 and NCT. Thus, education regarding concussive symptomatology, telemedicine evaluation and legislation must be implemented in concert with one another to create safe environments for athletes. Beyond the athletic setting, RTL policies aim to create a structured program of gradual reintroduction to the class environment that can minimize long-term disability while providing short-term accommodations.

\section{Limitations}

A few key limitations exist within the literature. Rural areas by country and locale each have unique features and challenges limiting generalizability. Literature remains scant on evaluation of specific rural disparities and their impact to health after mTBI/concussion. While many studies uncover 
risk factors associated with $\mathrm{mTBI} /$ concussions, few studies prospectively evaluate the effects of specific interventions on patient outcomes. Future studies should apply these findings to create context-specific best practices.

\section{Conclusions}

Rural patients are at unique risk for $\mathrm{mTBI} /$ concussions and health care costs. Barriers to care include lower socioeconomic status, longer distances to regional medical center, and decreased availability of neuroimaging and consultants. Due to socioeconomic and distance barriers, rural schools are less able to maintain personnel certified for concussion evaluation. Telemedicine is an emerging tool for remote triage and evaluation. Future studies applying mTBI management practices to specific rural locales are needed.

\section{Note}

The manuscript has been read and approved by all the authors and the requirements for authorship have been met by all authors. Each author believes that the manuscript represents honest work.

\section{Authors' Contributions}

J.K.Y.: concept and design of study; acquisition, review and interpretation of data; drafting and critically revising the manuscript for intellectual content; and approval of the submitted manuscript.

P.S.U.: acquisition, review and interpretation of data; drafting and critically revising the manuscript for intellectual content; approval of the submitted manuscript.

L.N.A.: acquisition, review and interpretation of data; drafting and critically revising the manuscript for intellectual content; and approval of the submitted manuscript.

R.R.L.P.: acquisition, review and interpretation of data; drafting and critically revising the manuscript for intellectual content; and approval of the submitted manuscript.

C.G.S.: acquisition, review and interpretation of data; drafting and critically revising the manuscript for intellectual content; and approval of the submitted manuscript.

T.A.C.: concept and design of study; acquisition, review and interpretation of data; drafting and critically revising the manuscript for intellectual content; approval of the submitted manuscript

\section{Funding}

None.

\section{Conflict of Interest}

None declared.

\section{References}

1 Galgano M, Toshkezi G, Qiu X, Russell T, Chin L, Zhao L-R. Traumatic brain injury: current treatment strategies and future endeavors. Cell Transplant 2017;26(7):1118-1130

2 Daneshvar DH, Nowinski CJ, McKee AC, Cantu RC. The epidemiology of sport-related concussion. Clin Sports Med 2011;30(1):1-17
3 Cancelliere C, Coronado VG, Taylor CA, Xu L. Epidemiology of isolated versus nonisolated mild traumatic brain injury treated in emergency departments in the United States, 20062012: sociodemographic characteristics. J Head Trauma Rehabil 2017;32(4):E37-E46

4 Laskowitz D, Grant G. Translational Research in Traumatic Brain Injury. United Kingdom: CRC Press; 2016

5 Feigin VL, Theadom A, Barker-Collo S, et al. BIONIC Study Group. Incidence of traumatic brain injury in New Zealand: a population-based study. Lancet Neurol 2013;12(1):53-64

6 Ratcliffe M, Burd C, Holder K, Fields A. Defining Rural at the U.S. Census Bureau. Available at: https://www.census.gov/ content/dam/Census/library/publications/2016/acs/acsgeo-1. pdf. Accessed May 2, 2019

7 du Plessis V, Beshiri R, Bollman RD. Definitions of "Rural." Available at: https://www150.statcan.gc.ca/n1/en/pub/21006-x/21-006-x2001003-eng.pdf?st=Cy17SoUn. Accessed October 19, 2019

8 Rural Indian. Available at: https://archive.india.gov.in/citizen/ graminbharat/graminbharat.php. Accessed May 2, 2019

9 Martin MF. Defining China's rural population. China Q 1992;130:392-401

$10 \mathrm{Ng}$ E, Wilkins R, Pole J, Adams OB. How far to the nearest physician. Rural and Small Town Canada Analysis Bulletin. 1999;1:1-7

11 Upadhyayula PS, Yue JK, Yang J, Birk HS, Ciacci JD. The current state of rural neurosurgical practice: an international perspective. J Neurosci Rural Pract 2018;9(1):123-131

12 Douthit N, Kiv S, Dwolatzky T, Biswas S. Exposing some important barriers to health care access in the rural USA. Public Health 2015;129(6):611-620

13 Boake C, McCauley SR, Pedroza C, Levin HS, Brown SA, Brundage SI. Lost productive work time after mild to moderate traumatic brain injury with and without hospitalization. Neurosurgery 2005;56(5):994-1003, discussion 994-1003

14 Yue JK, Burke JF, Upadhyayula PS, et al. Selective serotonin reuptake inhibitors for treating neurocognitive and neuropsychiatric disorders following traumatic brain injury: an evaluation of current evidence. Brain Sci 2017;7(8):93

15 Yue JK, Winkler EA, Sharma S, et al; the TRACK-TBI Investigators. Temporal profile of care following mild traumatic brain injury: predictors of hospital admission, follow-up referral and six-month outcome. Brain Inj 2017;31(13,14):1820-1829

16 Yue JK, Rick JW, Morrissey MR, et al; TRACK-TBI Investigators. Preinjury employment status as a risk factor for symptomatology and disability in mild traumatic brain injury: A TRACK-TBI analysis. NeuroRehabilitation 2018;43(2):169-182

17 Haarbauer-Krupa J, Taylor CA, Yue JK, et al. Screening for post-traumatic stress disorder in a civilian emergency department population with traumatic brain injury. J Neurotrauma 2017;34(1):50-58

18 McMahon P, Hricik A, Yue JK, et al. TRACK-TBI Investigators. Symptomatology and functional outcome in mild traumatic brain injury: results from the prospective TRACK-TBI study. J Neurotrauma 2014;31(1):26-33

19 Yue JK, Ngwenya LB, Upadhyayula PS, et al; TRACK-TBI Investigators. Emergency department blood alcohol level associates with injury factors and six-month outcome after uncomplicated mild traumatic brain injury. J Clin Neurosci 2017;45:293-298

20 Seabury SA, Gaudette É, Goldman DP, et al; TRACK-TBI Investigators. Assessment of follow-up care after emergency department presentation for mild traumatic brain injury and concussion: results from the TRACK-TBI Study. JAMA Netw Open 2018;1(1):e180210 
21 Stein MB, Jain S, Giacino JT, et al; and the TRACK-TBI Investigators. Risk of posttraumatic stress disorder and major depression in civilian patients after mild traumatic brain injury: a TRACK-TBI study. JAMA Psychiatry 2019;76(3):249-258

22 Kreitzer NP, Hart K, Lindsell CJ, et al. A comparison of satisfaction with life and the glasgow outcome scale-extended after traumatic brain injury: an analysis of the TRACK-TBI pilot study. J Head Trauma Rehabil 2019;34(3):E10-E17

23 Lavoie CA, Voaklander D, Beach JR, Gross DP. The association between rurality and return to work for workers' compensation claimants with work-related musculoskeletal injuries: An analysis of workers who failed to return to work within typical healing time frames. Int J Occup Med Environ Health 2017;30(5):715-729

24 Johnstone B, Price T, Bounds T, Schopp LH, Schootman M, Schumate D. Rural/urban differences in vocational outcomes for state vocational rehabilitation clients with TBI. NeuroRehabilitation 2003;18(3):197-203

25 Stewart TC, Gilliland J, Fraser DD. An epidemiologic profile of pediatric concussions: identifying urban and rural differences. J Trauma Acute Care Surg 2014;76(3):736-742

26 Karwat ID, Krupa S, Gorczyca R. Causes and consequences of head injuries among rural inhabitants hospitalised in a multi-organ injury Ward. II. Circumstances, types and consequences of head injuries. Ann Agric Environ Med 2009;16(1):23-29

27 Munivenkatappa A, Devi BI, Gregor TI, Bhat DI, Kumarsamy AD, Shukla DP. Bicycle accident-related head injuries in India. J Neurosci Rural Pract 2013;4(3):262-266

28 Zhao YD, Wang W. Neurosurgical trauma in People's Republic of China. World J Surg 2001;25(9):1202-1204

29 Yang J, Phillips G, Xiang H, Allareddy V, Heiden E, Peek-Asa C. Hospitalisations for sport-related concussions in US children aged 5 to 18 years during 2000-2004. Br J Sports Med 2008;42(8):664-669

30 Bernard CO, Ponsford JA, McKinlay A, McKenzie D, Krieser D. Predictors of post-concussive symptoms in young children: injury versus non-injury related factors. J Int Neuropsychol Soc 2016;22(8):793-803

31 Brown AM, Twomey DM, Wong Shee A. Evaluating mild traumatic brain injury management at a regional emergency department. Inj Prev 2018;24(5):390-394

32 Levy AS, Orlando A, Salottolo K, Mains CW, Bar-Or D. Outcomes of a nontransfer protocol for mild traumatic brain injury with abnormal head computed tomography in a rural hospital setting. World Neurosurg 2014;82(1-2):e319-e323

33 Schootman M, Fuortes LJ. Ambulatory care for traumatic brain injuries in the US, 1995-1997. Brain Inj 2000;14(4):373-381

34 Graves JM, Mackelprang JL, Moore M, et al. Rural-urban disparities in health care costs and health service utilization following pediatric mild traumatic brain injury. Health Serv Res 2019;54(2):337-345

35 Zanaboni P, Wootton R. Adoption of routine telemedicine in Norwegian hospitals: progress over 5 years. BMC Health Serv Res 2016;16:496

36 Vargas BB, Channer DD, Dodick DW, Demaerschalk BM. Teleconcussion: an innovative approach to screening, diagnosis, and management of mild traumatic brain injury. Telemed J E Health 2012;18(10):803-806

37 Tsai J, Whealin JM, Scott JC, Harpaz-Rotem I, Pietrzak RH. Examining the relation between combat-related concussion, a novel 5-factor model of posttraumatic stress symptoms, and health-related quality of life in Iraq and Afghanistan veterans. J Clin Psychiatry 2012;73(8):1110-1118

38 Whealin JM, Stotzer RL, Pietrzak RH, et al. Deployment-related sequelae and treatment utilization in rural and urban war veterans in Hawaii. Psychol Serv 2014;11(1):114-123

39 Casa DJ, Almquist J, Anderson SA, et al. The inter-association task force for preventing sudden death in secondary school athletics programs: best-practices recommendations. J Athl Train 2013;48(4):546-553

40 Meehan WP III, Mannix R. A substantial proportion of life-threatening injuries are sport-related. Pediatr Emerg Care 2013;29(5):624-627

41 Pryor RR, Casa DJ, Vandermark LW, et al. Athletic training services in public secondary schools: a benchmark study. J Ath Train 2015;50(2):156-162

42 Kroshus E, Rivara FP, Whitlock KB, Herring SA, Chrisman SPD. Disparities in athletic trainer staffing in secondary school sport: implications for concussion identification. Clin J Sport Med 2017;27(6):542-547

43 McGrath SP, McGrath ML, Bastola D. Developing a concussion assessment mHealth app for certified Athletic Trainers. AMIA Annu Symp Proc 2018;2017:1282-1291

44 Murphy A, Kaufman MS, Molton I, Coppel DB, Benson J, Herring SA. Concussion evaluation methods among Washington State high school football coaches and athletic trainers. PM R 2012;4(6):419-426

45 Rivara FP, Schiff MA, Chrisman SP, Chung SK, Ellenbogen RG, Herring SA. The effect of coach education on reporting of concussions among high school athletes after passage of a concussion law. Am J Sports Med 2014;42(5):1197-1203

46 Chrisman SP, Schiff MA, Chung SK, Herring SA, Rivara FP. Implementation of concussion legislation and extent of concussion education for athletes, parents, and coaches in Washington State. Am J Sports Med 2014;42(5):1190-1196

47 Lyons VH, Moore M, Guiney R, et al. Strategies to address unmet needs and facilitate return to learn guideline adoption following concussion. J Sch Health 2017;87(6):416-426

48 Jagoda AS, Bazarian JJ, Bruns JJ Jr, et al. Clinical policy: neuroimaging and decisionmaking in adult mild traumatic brain injury in the acute setting. J Emerg Nurs 2009;35(2):e5-e40

49 Stiell IG, Wells GA, Vandemheen $\mathrm{K}$, et al. The Canadian CT head rule for patients with minor head injury. Lancet 2001;357(9266):1391-1396

50 Foks KA, van den Brand CL, Lingsma HF, et al. External validation of computed tomography decision rules for minor head injury: prospective, multicentre cohort study in the Netherlands. BMJ 2018;362:k3527

51 Villarejo D, Baron SL. The occupational health status of hired farm workers. Occup Med 1999;14(3):613-635 